\section{MAPping out aneurysm}

\section{By Lev Osherovich, Senior Writer}

A team from The Johns Hopkins University School of Medicine has uncovered a role for the MAP kinase pathway in aortic aneurysms caused by the genetic disorder Marfan syndrome. The findings could open up a new indication for inhibitors of the pathway already in the clinic for cancer, but it is unclear whether hitting the pathway will be useful for nonhereditary forms of aortic aneurysms.

Marfan syndrome affects up to 1 in 5,000 people and is caused by a mutation in the fibrillin 1 (FBN1) gene that leads to abnormal connective tissue throughout the body. The most serious clinical manifestation of the disorder is aneurysm due to weakening of the connective tissue of the aorta.

Surgery is the standard care for aortic aneurysms, but undiagnosed aneurysms can burst and cause life-threatening hemorrhages. There is no way to prevent aneurysms from developing in susceptible patients.

Now, a team led by Harry Dietz, professor of medicine, pediatric cardiology and genetics at Johns Hopkins and an investigator at the Howard Hughes Medical Institute, has evidence that blocking MAP kinase signaling could help prevent aortic aneurysm. ${ }^{1,2}$

The pathway involves a cascade of intracellular kinases that relays signals from the cell surface into the nucleus. It is often hyperactive in a range of cancer and autoimmune indications, and at least a dozen companies have clinical compounds that hit components of the MAP kinase pathway to treat cancer and autoimmune disease.

Dietz's team characterized mice with mutations in Fbn1 that mimic Marfan syndrome and found that the MAP kinase pathway mediates the effects of two proteins implicated in aneurysm - the vasoconstrictive peptide hormone angiotensin II and transforming growth factor- $\beta$ (TGFB).

"A few years back, we came to the realization that TGFB signaling in general was important in aneurysm, as is angiotensin signaling," said Dietz. The new work identifies the downstream effects of these extracellular factors.

The findings were reported in back-to-back papers in Science.

\section{Many kinases}

Drawing on prior evidence of the MAP kinase pathway's involvement in TGFB signaling in cancer and inflammation, Dietz's team looked at phosphorylation patterns of MAP kinase pathway components in mouse models of Marfan syndrome and found evidence of excessive activity.

The abnormally active MAP kinase pathway components were MAP kinase 3 (Mapk3; Erk-1), Mapk1 (Erk-2) and MAP kinase kinase 1 (Map2k1; Mek1).

Blocking this pathway with BAY 86-9766, which targets MEK1 and a related kinase, MAP2K2 (MEK2), lowered abnormal aortic root growth in mice compared with using vehicle. BAY 86-9766, from Ardea Biosciences Inc. and partner Bayer AG, is in Phase I and Phase II testing for solid tumors.

Dietz's team also found that pharmacological blockade of another MAP kinase pathway component-c-jun N-terminal kinase 1 (Jnk1; Mapk8)-decreased abnormal aortic growth and increased survival of Marfan mice compared with vehicle-treated controls.

\section{Angiotensin tamer}

In the second study, Dietz's team looked at how angiotensin II type 1 receptor (Agtr1) and Agtr2 affected MAP kinase pathway activation and aortic pathology in Marfan mice.

The team found that blocking production of angiotensin II with the generic angiotensin-converting enzyme (ACE) inhibitor enalapril, which reduces the activity of both AGTR1 and AGTR2, did not significantly alter the pathology of Marfan syndrome in mice.

However, knocking out Agtr2 while leaving Agtr1 intact decreased aortic root growth and improved survival of Marfan mice compared with controls that had intact Agtr2.

To Dietz, these data suggest that AGTR1 has a protective role against aneurysm, whereas AGTR2 has the opposite effect. Indeed, Marfan mice treated with the generic AGTR2 antagonist losartan fared better than vehicle-treated controls or mice treated with enalapril.

The team also found that losartan lowered ERK-1 and ERK-2 activity compared with vehicle control.

Dietz is running a Phase II trial of losartan to treat Marfan syndromeassociated aortic aneurysm.

\section{Aortadoxy}

Dietz's studies offer an array of therapeutic targets for Marfan syndromerelated aortic aneurysm, but homing in on the best targets will require further preclinical work.

At first blush, directly blocking angiotensin II or TGFB seems like the most straightforward approach, but Dietz noted that those targets are not appropriate for all patients.

Dietz said losartan and other angiotensin receptor blockers (ARBs) are not suitable for pediatric use and are not effective in some adults, whereas targeting TGFB could have side effects.

"Some people can't tolerate high doses of ARBs," said Dietz. "TGFB can suppress inflammation and can act as a tumor suppressor."

Instead, Dietz thinks that hitting the MAP kinase pathway may be a better approach, though which specific target to focus on is still up in the air.

It's unclear, however, if BAY 86-9766 would be well tolerated in an aneurysm prevention regimen.

Karl Ziegelbauer, head of Bayer's oncology therapeutic research group, told SciBX that Dietz's BAY 86-9766 dosing regimen is "close to or at the maximum tolerated dose in preclinical tumor experiments" with the compound. 


\section{ANALYSIS}

\section{TARGETS \& MECHANISMS}

Thus, Ziegelbauer said, the therapeutic window for using BAY 86-9766 in noncancer indications could be narrow, especially if it were a chronic treatment.

The most advanced trial of BAY 86-9766 is a Phase II study in combination with Nexavar sorafenib for liver cancer. Nexavar, a kinase inhibitor with multiple targets, is comarketed by Bayer and Onyx Pharmaceuticals Inc. to treat liver and renal cancer.

Dietz said his team used BAY 86-9766 primarily because it was readily available, so it's possible that hitting other kinases in the pathway besides MEK1 and MEK2 could also prevent aortic aneurysm.

Thus, identifying the most proximal MAP kinase pathway target could allow the team to use a more appropriate compound than BAY 86-9766.

"More needs to be learned about the effects of inhibiting the MAP kinase pathway," said Dietz. "The concept is that as we drill deeper into the pathway, the closer we will get to the disease-initiating event and the less side effects there will be."

Dietz next plans to work with NIH's Therapeutics for Rare and Neglected Diseases (TREND) program to test more selective MAP kinase pathway inhibitors in Marfan syndrome mice.

\section{Going broad}

In addition to picking the best target, another outstanding question is whether TGFB, angiotensin II and the MAP kinase pathway are relevant to the nonhereditary aortic aneurysms that strike many middle-aged patients.

"While aneurysm in the general population is a complex trait, the emerging view is that [TGFB] is going to be important in aneurysm in general," said Dietz.

Ziad Mallat, professor of cardiovascular medicine at the University of Cambridge, cautioned that the specific role of TGFB in Marfan syndrome may be different from its role in other forms of aneurysm.
Last year, Mallat's team reported that $\mathrm{mAb}$ blockade of TGFB in a mouse model of nonMarfan aneurysm actually exacerbated disease compared with no treatment. ${ }^{3}$

Thus, he suggested further experiments "inhibiting downstream signaling pathways" of TGFB in different models of aneurysm formation in addition to Marfan mice in order to see whether TGFB is relevant in other models.

Dietz countered that Mallat's study was done in a mouse model with very severe and rapidly developing aneurysm and may not accurately reflect clinical conditions. He cited evidence that improper regulation of TGFB has turned up in bicuspid aortic valve disease, another cause of aortic aneurysm.

He has filed for patents covering the use of MAP kinase pathway inhibitors to treat aortic aneurysm.

Osherovich, L. SciBX 4(17); doi:10.1038/scibx.2011.473

Published online April 28, 2011

REFERENCES

1. Holm, T.M. et al. Science; published online April 15, 2011; doi:10.1126/science.1192149

Contact: Harry C. Dietz, The Johns Hopkins University School of Medicine, Baltimore, Md. e-mail: hdietz@jhmi.edu

2. Habashi, J.P. et al. Science; published online April 15, 2011; doi:10.1126/science.1192152

Contact: Harry C. Dietz, The Johns Hopkins University School of Medicine, Baltimore, Md. e-mail: hdietz@jhmi.edu

3. Wang, Y. et al. J. Clin. Invest. 120, 422-432 (2010)

\section{COMPANIES AND INSTITUTIONS MENTIONED}

Ardea Biosciences Inc. (NASDAQ:RDEA), San Diego, Calif. Bayer AG (Xetra:BAY), Leverkusen, Germany The Johns Hopkins University School of Medicine, Baltimore, Md. Howard Hughes Medical Institute, Chevy Chase, Md. National Institutes of Health, Bethesda, Md. Onyx Pharmaceuticals Inc. (NASDAQ:ONXX), Emeryville, Calif. University of Cambridge, Cambridge, U.K. 